\title{
Traffic Control Problems using Graph Connectivity
}

\author{
Arun Kumar Baruah \\ Department of Mathematics, Dibrugarh University \\ Dibrugarh: 786004, Assam, India
}

\begin{abstract}
In modern day automobile traffic the problem of traffic congestion calls for the design of efficient control strategies. In this paper it is argued that in order to have efficient and systematic solutions to a traffic control problem at an intersection, graph theoretic models of the problem are quite appropriate for its exploitation. Connectivity of compatibility graph of a traffic intersection can be used to study the most efficient route or the traffic control system to direct the traffic flow to its maximum capacity using the minimum number of edges or the minimum number of vertices. This result has got application in traffic control problems at an arbitrary intersection so as to minimize the waiting time of the traffic participants and the cost of locating the sensors in order to collect traffic data.
\end{abstract}

\section{Keywords}

Compatibility Graph, Edge Connectivity, Intelligent Transportation System, Traffic Control, Traffic Sensors, Vertex Connectivity.

\section{INTRODUCTION}

Traffic theory is a physical phenomenon that aims at understanding and improving automobile traffic, and the problem associated with it such as traffic congestion [1]. The traffic control problem is to minimize the waiting time of the public transportation while maintaining the individual traffic flow optimally [2]. Significant development of traffic control systems using traffic lights has been achieved since the first traffic controller was installed in London in 1868. The first green wave was realised in Salt Lake City (U.K.) in 1918, and the first area traffic controller was introduced in Toronto in 1960. At the beginning, electromechanical devices were used to perform traffic control. Then Intelligent Transportation System (ITS) is used extensively in urban areas to control traffic at an intersection [3]. The traffic data in a particular region can be used to direct the traffic flow to improve traffic output without adding new roads. In order to collect accurate traffic data semi conductor-based controllers known as sensors were placed in different places to collect traffic information are used in traffic control system [3], [4], [5]. Nowadays, microprocessor based controller are used in Traffic Control Systems. The combinatorial approach to the optimal traffic control problem was founded by Stoffers [6] in 1968 by introducing the Compatibility Graph of traffic streams.

One of the main uses of traffic theory is the development of traffic models which can be used for estimation, prediction, and control related tasks for the automobile traffic process. In this paper graph theoretic model of a traffic control problem at an intersection is used for its solution. The resulting compatibility graph of the intersection and its connectivity is used to study the most efficient route or the traffic control system to direct the traffic flow to its maximum capacity using the minimum number of edges or the minimum number of vertices.

\section{INTELLIGENT TRANSPORTATION SYSTEM}

The term Intelligent Transportation System (ITS) refers to information and communication technology applied to transport infrastructure and vehicles, that improves transport outcomes such as transport safety, transport productivity, transport reliability, informed traveller choice, environmental performance etc. [7], [8].

ITS mainly comes from the problems caused by traffic congestion and synergy of new information technology for simulation, real time control and communication networks. Traffic congestion has been increased world wide as a result of increased motorization, urbanization, population growth and changes in population density. Congestion reduces efficiency of transportation infrastructure and increases travel time, air pollution and fuel consumption.

At the beginning of 1920, in United States large increase in both motorization and urbanization led to the migration of the population from sparsely populated rural areas and densely packed urban areas into suburbs (sub urban areas). Recent governmental activity in the area of ITS specially in the United States is motivated by an increased focus on homeland security. Other parts of the developing world, such as China, remain largely rural but are rapidly urbanizing and industrializing. The urban infrastructure is being rapidly developing, providing an opportunity to build new systems that incorporate ITS at early stage.

Intelligent Transport Systems vary in technologies applied, from basic management system such as car navigation; traffic signal control systems; container management system; variable message sign; automatic number plate recognition or speed cameras to monitor applications; such as security CCTV systems; and to more advanced applications that integrate live data and feedback from a number of other sources, such as parking guidance and information systems; weather information etc. Additional predictive techniques are being developed to allow advanced modeling and comparison with historical data. The traffic flow predictions will be delivered to the drivers via different channels such as roadside billboards, radio stations, internet, and on vehicle GPS (Global Positioning Systems) systems. One of the components of an ITS is the live traffic data collection. To collect accurate traffic data sensors have to be placed on the roads and streets to measure the flow of traffic. Some of the constituent technologies implemented in ITS are namely, Wireless Communication, Computational technologies, Sensing technologies, Video Vehicle Detection etc. Sensing technologies, which is the present interest, mean briefly the following:

The technological advances in telecommunication and information technology, coupled with microchip, RFID ( Radio Frequency Identification), and inexpensive intelligent beacon sensing technologies, have enhanced the technical capabilities that will facilitate safety of the traffic participants for intelligent transportation system globally. Sensing systems for ITS are vehicle- and infrastructure based 
networked systems i.e. Intelligent vehicle technologies. Infrastructure sensors are such as in road reflector devices that are already installed or embedded in the road or surrounding the road e.g. on building, posts and signs, as required and may be manually disseminated during preventive road construction maintenance or by sensor injection machinery for rapid development [9].

\section{COMPATIBILITY GRAPH AND ITS CONNECTIVITY}

Vehicles approaching an intersection prepare themselves to perform a certain maneuver i.e. to drive through, turn left, or turn right at an intersection. The vehicles that perform this maneuver represent a flow component. Such an arrival flow component is called a traffic stream [10]. For solving the control problem it is necessary to know relations between traffic streams at an intersection. Traffic streams mean briefly the following :

Traffic streams $\boldsymbol{\sigma}_{\mathbf{i}}$ on an intersection are elements of the set of traffic stream, $\boldsymbol{\sigma}$ i.e

$$
\sigma=\left(\sigma_{1}, \sigma_{2}, \sigma_{3}, \ldots, \sigma_{i}\right)
$$

where $\sigma_{1}, \sigma_{2}, \sigma_{3}, \ldots, \sigma_{i}$ are the individual traffic streams of an intersection.

To study the traffic control problem at an arbitrary intersection, it has to be modeled mathematically by using a simple graph for the traffic collection data problem. The set of edges of the underlying graph will represent the communication link between the set of nodes i.e. traffic streams at an intersection. In the graph representing the traffic control problem, the traffic streams which can move simultaneously at an intersection without any conflict will be joined by an edge and the streams which cannot move together will not be connected by an edge. The graph obtained thus is a connected graph and will be referred as the compatibility graph of the intersection corresponding to the traffic control problem. In order to define a cut-set and the connectivity of the compatibility graph, the underlying graph $G$ considered as $G=(V, E)$ where $V(G)$ denotes the set of vertices of $G$ and $E(G)$ denotes the set of edges of $G$. A cutset $F$ is a set of edges whose removal from $G$ leaves $G$ disconnected. Also it results in the increase in the number of components of $G$ by one. Each cut-set of the compatibility graph $G$ consists of a certain number of edges. The number of edges in the smallest cut-set i.e. the cut-set with fewest number of edges is defined as the edge connectivity of $G$. The vertex connectivity of the compatibility graph $G$ is defined as the minimum number of vertices whose removal from $G$ leaves the remaining graph disconnected. Detail account in these aspects may be found in [11], [12], [13]. The following theorems are due to [11].

\subsection{Theorem}

The edge connectivity of a graph $G$ cannot exceed the degree of the vertex with the smallest degree in $G$.

\subsection{Theorem}

The vertex connectivity of any graph $G$ can never exceed the edge connectivity of $G$.

\subsection{Theorem}

The maximum vertex connectivity one can achieve with a graph $G$ of $n$ vertices and $e$ edges $(e \geq n-1)$ is the integral part of the number $2 e / n$; i.e., $\lfloor 2 e / n\rfloor$
The results of the Theorems 3.1, 3.2, 3.3 can be summarized as below :

vertex connectivity $\leq$ edge connectivity $\leq 2 e / n$

maximum vertex connectivity possible $=\lfloor 2 e / n\rfloor$

The traffic sensors can be placed on each edge in a cut-set of $G$ determined by its edge connectivity as well as on each vertex of $G$ determined by its vertex connectivity. These sensors will provide complete traffic information for the control system. Thus optimal locations for the traffic sensors can be obtained by using edge connectivity and vertex connectivity of the compatibility graph $G$. Relevant to this aspect for the graph $G$, algorithm for finding edge control set, which is a cut-set, and which completely determines every flow of $G$ may be found in [14].

\section{AN EXAMPLE}

The example considered is a traffic control problem at a four leg intersection, with five streams as shown in Fig. 1 and the corresponding compatibility graph as shown in Fig. 2 below:

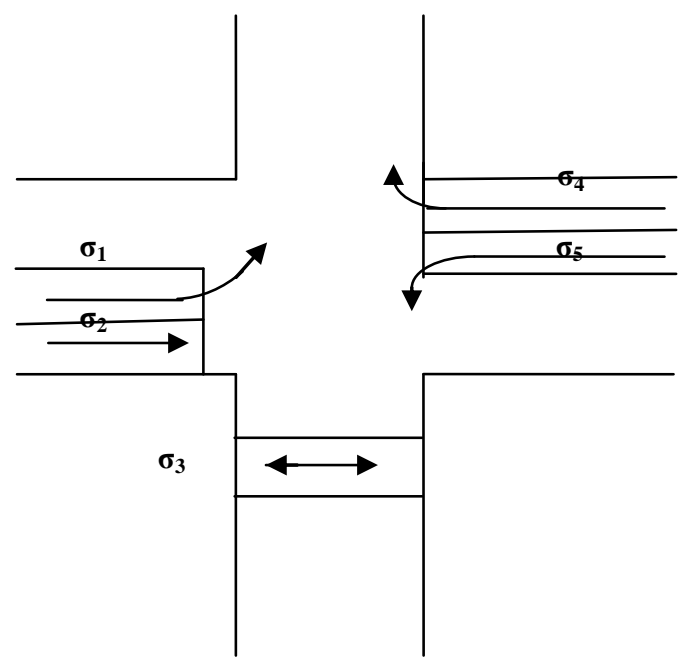

Fig. 1 : An intersection with five traffic streams

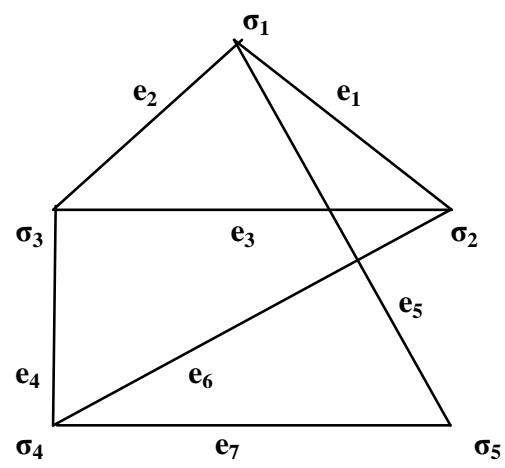

Fig. 2 : Compatibility graph representing the above intersection (Fig. 1)

In the compatibility graph (Fig. 2), number of vertices $n$ is 5 and number of edges $e$ is 7. Following the discussion in Section 3, vertex connectivity as well as edge connectivity which can be achieved here are as high as 2 . It is not difficult to find edges $\mathbf{e}_{5}$ and $\mathbf{e}_{7}$ corresponding to the edge connectivity as well as vertices $\sigma_{1}$ and $\sigma_{4}$ corresponding to 
the vertex connectivity whose removal disconnects the compatibility graph. The disconnected graphs having two components obtained after removing the edges $\mathbf{e}_{5}$ and $\mathbf{e}_{7}$ as well as vertices $\sigma_{1}$ and $\sigma_{4}$ from the compatibility graph are as shown in the Fig. 3 and Fig. 4 respectively as below:

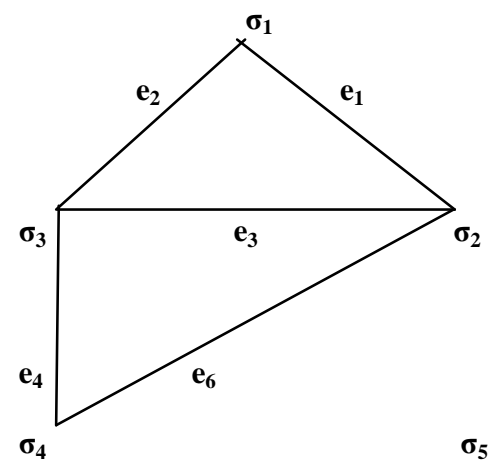

Fig. 3 : Graph obtained after removal of the edges $e_{5}$ and $\mathbf{e}_{7}$

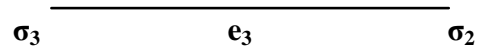

$\sigma_{5}$

Fig. 4 : Graph obtained after removal of the vertices $\sigma_{1}$ and $\sigma_{4}$

From the above discussion it is clear that sensors have to placed either in the edges $\mathbf{e}_{5}$ and $\mathbf{e}_{7}$ or in the vertices $\sigma_{1}$ and $\sigma_{4}$, which will provide complete information and can be delivered to the traffic participants, regarding traffic flow, congestion etc.

\section{APPLICATIONS}

Edge connectivity and vertex connectivity have wide application in traffic control problems at an intersection. The edges given by the edge connectivity and the vertices given by the vertex connectivity determine the exact locations where the sensors have to be placed which minimizes the total cost and the complete data of the traffic problem can be obtained by using either of the two connectivities.

The connectivities are of great importance in studying properties of communication and transportation networks, as it is necessary to know the maximum rate of flow that is possible from one node to another in the network. Also there are applications in many other networks such as network representing roads with traffic capacities, or link in a computer network with data transmission capacities, or currents in a electric network or some application in industrial settings etc.

\section{CONCLUSION}

In this paper edge connectivity as well as vertex connectivity are used as graph theoretic tools to study traffic control problem at an intersection. As the edges of the edge connectivity represents the flow of traffic at an intersection, the waiting time of the traffic participants can be minimized by controlling the edges of the edge connectivity. This can be achieved by placing traffic sensors on each such edges of the edge connectivity of the transportation network which will provide complete traffic information of the network. As an alternative to above, sensors can also be placed on each vertex of the vertex connectivity of the transportation network for getting complete traffic information of the network. Although comparatively less number of streams is considered in the example, the idea can be generalised and can be used to study traffic control problem at any intersection with more number of traffic streams.

\section{ACKNOWLEDGEMENTS}

This Research is funded by grants from the UGC, New Delhi, India as a Major Research Project awarded to the author.

\section{REFERENCES}

[1] Gazis, D. C., 1970, Traffic Science, John Wiley \& Sons, New York.

[2] Gazis, D. C., 2002, Traffic Theory, Kluwer Academic Publishers, London.

[3] Gu, W., Jia, X., 2005, On a Traffic Control Problem, $8^{\text {th }}$ International Symposium on Parallel Architectures, Algorithms and Networks (ISPAN' 05).

[4] Gu, W., Jia, X., 2006, On a Traffic Sensing Problem, Preprint, Texas State University, 2006.

[5] Riedel, T., 2002, Traffic Control using Graph Theory, Verkehrs- Systeme AG.

[6] Stoffers, E. K., 1968, Scheduling of Traffic Lights - A New Approach, Transportation Research, 2, 199.

[7] Monahan, Torin, 2007, “War Rooms" of the street: Suveillance Practices in Transportation Control Centers. The Communication Review 10(4), 367-389.

[8] World Road Association, 2002, Road Network Operation Handbook.

[9] Tarnoff, P., John, B., Darcy, M., Young, S. E. et. al., 2009, Continuing Evolution of Travel Data Information Collection and Processing, Transportation Research Board Annual Meeting.

[10] Guberinic, S., Senborn, G., Lazic, B.. 2008, Optimal Traffic Control Urban Intersection, CRC Press.

[11] Deo, N., 2000, Graph Theory with Applications to Engineering and Computer Science, Prentice Hall Inc., New Jersey.

[12] West, D. B., 2001, Introduction to Graph Theory, Prentice Hall Inc., New Jersey.

[13] Wilson, R. J., Watkins, J. J., 1989, Graph : An Introductory Approach, John Wiley \& Sons, New York.

[14] Baruah, N., Baruah, A. K., 2012, On a Traffic Control Problem using Cut-Set of Graph, International Journal of Advanced Networking and Applications, 3(4), 12401244. 